\title{
Integral type contractions in modular metric spaces
}

\author{
Bahareh Azadifar ${ }^{1}$, Ghadir Sadeghi ${ }^{1}$, Reza Saadati $^{2}$ and Choonkil Park ${ }^{3 *}$
}

${ }^{\text {*Correspondence: }}$

baak@hanyang.ac.kr

${ }^{3}$ Department of Mathematics, Research Institute for Natural

Sciences, Hanyang University, Seoul, 133-791, Korea

Full list of author information is

available at the end of the article

\begin{abstract}
We prove the existence and uniqueness of a common fixed point of compatible mappings of integral type in modular metric spaces.
\end{abstract}

MSC: Primary 47H09; 47H10; secondary 46A80

Keywords: modular metric space; compatible maps; contractive inequality of integral type; fixed point

\section{Introduction and preliminaries}

The metric fixed point theory is very important and useful in mathematics. It can be applied in various branches of mathematics, variational inequalities optimization and approximation theory. In 1976, Jungck [1] proved a common fixed point theorem for commuting maps generalizing the Banach contraction mapping principle. This result was further generalized and extended in various ways by many authors. On the other hand, Sessa [2] defined weak commutativity as follows.

Let $(X, d)$ be a metric space, the self-mappings $f, g$ are said to be weakly commuting if $d(f g(x), g f(x)) \leq d(g(x), f(x))$ for all $x \in X$. Further, Jungck [3] introduced more generalized commutativity, the so-called compatibility, which is more general than weak commutativity. Let $f, g$ be self-mappings of a metric space $(X, d)$. The mappings $f$ and $g$ are said to be compatible if $\lim _{n \rightarrow \infty} d\left(f g\left(x_{n}\right), g f\left(x_{n}\right)\right)=0$, whenever $\left\{x_{n}\right\}_{n=1}^{\infty}$ is a sequence in $X$ such that $\lim _{n \rightarrow \infty} f\left(x_{n}\right)=\lim _{n \rightarrow \infty} g\left(x_{n}\right)=z$ for some $z \in X$. Clearly, weakly commuting mappings are compatible, but neither implication is reversible. Let $X=[0,1)$ with the usual metric. We define mappings $f$ and $g$ on $X$ by

$$
f(x):=\left\{\begin{array}{ll}
\frac{2}{3} & \text { if } 0 \leq x<\frac{2}{3}, \\
1-\frac{x}{2} & \text { if } \frac{2}{3} \leq x<1
\end{array} \text { and } g(x):= \begin{cases}\frac{2}{3} & \text { if } 0 \leq x<\frac{2}{3} \\
\frac{4}{3}-x & \text { if } \frac{2}{3} \leq x<1\end{cases}\right.
$$

Let $\left\{x_{n}\right\}_{n=1}^{\infty}$ be a sequence in $X$ with $\lim _{n \rightarrow \infty} f\left(x_{n}\right)=\lim _{n \rightarrow \infty} g\left(x_{n}\right)=z$, then $z=\frac{2}{3}$ and $\lim _{n \rightarrow \infty} f g\left(x_{n}\right)=\lim _{n \rightarrow \infty} g f\left(x_{n}\right)=\frac{2}{3}$. Thus the pair $(f, g)$ is compatible on $X$. In [4] Branciari obtained a fixed point theorem for a single mapping satisfying an analogue of the Banach contraction principle for integral type inequality (see also [5-7]). Vijayaraju et al. [8] proved the existence of the unique common fixed point theorem for a pair of maps satisfying a general contractive condition of integral type. Recently, Razani and Moradi [9] proved the common fixed point theorem of integral type in modular spaces. The purpose of this paper is to generalize and improve Jungck's fixed point theorem [3] and Branciari's

@2013 Azadifar et al.; licensee Springer. This is an Open Access article distributed under the terms of the Creative Commons Attribution License (http://creativecommons.org/licenses/by/2.0), which permits unrestricted use, distribution, and reproduction in any medium, provided the original work is properly cited. 
result [4] to compatible maps in metric modular spaces. The notions of a metric modular on an arbitrary set and the corresponding modular space, more general than a metric space, were introduced and studied recently by Chistyakov [10]. In the sequel, we recall some basic concepts about modular metric spaces.

Definition 1.1 A function $\omega:(0, \infty) \times X \times X \rightarrow[0, \infty]$ is said to be a metric modular on $X$ if it satisfies the following three axioms:

(i) given $x, y \in X, \omega_{\lambda}(x, y)=0$ for all $\lambda>0$ if and only if $x=y$;

(ii) $\omega_{\lambda}(x, y)=\omega_{\lambda}(y, x)$ for all $\lambda>0$ and $x, y \in X$;

(iii) $\omega_{\lambda+\mu}(x, y) \leq \omega_{\lambda}(x, z)+\omega_{\mu}(z, y)$ for all $\lambda, \mu>0$ and $x, y, z \in X$.

If, instead of (i), we have only the condition (i) ${ }^{\prime} \omega_{\lambda}(x, x)=0$ for all $\lambda>0$ and $x \in X$, then $\omega$ is said to be a (metric) pseudo-modular on $X$. The main property of a (pseudo)modular $\omega$ on a set $X$ is the following: given $x, y \in X$, the function $0<\lambda \mapsto \omega_{\lambda}(x, y) \in[0, \infty]$ is nonincreasing on $(0, \infty)$. In fact, if $0<\mu<\lambda$, then (iii), (i)' and (ii) imply

$$
\omega_{\lambda}(x, y) \leq \omega_{\lambda-\mu}(x, x)+\omega_{\mu}(x, y)=\omega_{\mu}(x, y)
$$

for all $x, y \in X$. If follows that at each point $\lambda>0$ the right limit $\omega_{\lambda+0}(x, y):=\lim _{\varepsilon \rightarrow+0} \omega_{\lambda+\varepsilon}(x$, $y)$ and the left limit $\omega_{\lambda-0}(x, y):=\lim _{\varepsilon \rightarrow+0} \omega_{\lambda-\varepsilon}(x, y)$ exist in $[0, \infty]$ and the following two inequalities hold:

$$
\omega_{\lambda+0}(x, y) \leq \omega_{\lambda}(x, y) \leq \omega_{\lambda-0}(x, y)
$$

for all $x, y \in X$. We know that if $x_{0} \in X$, the set $X_{\omega}=\left\{x \in X: \lim _{\lambda \rightarrow \infty} \omega_{\lambda}\left(x, x_{0}\right)=0\right\}$ is a metric space, called a modular space, whose metric is given by

$$
d_{\omega}^{0}(x, y)=\inf \left\{\lambda>0: \omega_{\lambda}(x, y) \leq \lambda\right\}
$$

for all $x, y \in X_{\omega}$. We know that (see [10]) if $X$ is a real linear space, $\rho: X \rightarrow[0, \infty]$ and

$$
\omega_{\lambda}(x, y)=\rho\left(\frac{x-y}{\lambda}\right)
$$

for all $\lambda>0$ and $x, y \in X$, then $\rho$ is modular on $X$ if and only if $\omega$ is metric modular on $X$.

Example 1.2 The following indexed objects $\omega$ are simple examples of (pseudo)modulars on a set $X$. Let $\lambda>0$ and $x, y \in X$, we have:

(a) $\omega_{\lambda}^{a}(x, y)=\infty$ if $x \neq y, \omega_{\lambda}^{a}(x, y)=0$ if $x=y$;

and if $(X, d)$ is a (pseudo)metric space with (pseudo)metric $d$, then we also have:

(b) $\omega_{\lambda}^{b}(x, y)=\frac{d(x, y)}{\varphi(\lambda)}$, where $\varphi:(0, \infty) \rightarrow(0, \infty)$ is a nondecreasing function;

(c) $\omega_{\lambda}^{c}(x, y)=\infty$ if $\lambda \leq d(x, y)$, and $\omega_{\lambda}^{c}(x, y)=0$ if $\lambda>d(x, y)$;

(d) $\omega_{\lambda}^{d}(x, y)=\infty$ if $\lambda<d(x, y)$, and $\omega_{\lambda}^{d}(x, y)=0$ if $\lambda \geq d(x, y)$.

Definition 1.3 Let $X_{\omega}$ be a modular metric space.

(1) The sequence $\left\{x_{n}\right\}_{n=1}^{\infty}$ in $X_{\omega}$ is said to be convergent to $x \in X_{\omega}$ if $\omega_{\lambda}\left(x_{n}, x\right) \rightarrow 0$ as $n \rightarrow \infty$ for all $\lambda>0$.

(2) The sequence $\left\{x_{n}\right\}_{n=1}^{\infty}$ in $X_{\omega}$ is said to be Cauchy to $x \in X_{\omega}$ if $\omega_{\lambda}\left(x_{n}, x_{m}\right) \rightarrow 0$ as $m, n \rightarrow$ $\infty$ for all $\lambda>0$. 
(3) A subset $C$ of $X_{\omega}$ is said to be closed if the limit of a convergent sequence of $C$ always belongs to $C$.

(4) A subset $C$ of $X_{\omega}$ is said to be complete if any Cauchy sequence of $C$ is a convergent sequence and its limit is in $C$.

(5) A subset $C$ of $X_{\omega}$ is said to be bounded if for all $\lambda>0, \delta_{\omega}(C)=\sup \left\{\omega_{\lambda}(x, y) ; x, y \in\right.$ $C\}<\infty$.

\section{A common fixed point theorem for contractive condition maps}

Here, the existence of a common fixed point for $\omega$-compatible mappings satisfying a contractive condition of integral type in modular metric spaces is studied. We recall the following definition.

Definition 2.1 Let $X_{\omega}$ be a modular metric space induced by metric modular $\omega$. Two selfmappings $T, h$ of $X_{\omega}$ are called $\omega$-compatible if $\omega_{\lambda}\left(T h x_{n}, h T x_{n}\right) \rightarrow 0$, whenever $\left\{x_{n}\right\}_{n=1}^{\infty}$ is a sequence in $X_{\omega}$ such that $h x_{n} \rightarrow z$ and $T x_{n} \rightarrow z$ for some point $z \in X_{\omega}$ and for $\lambda>0$.

Theorem 2.2 Let $X_{\omega}$ be a complete modular metric space. Suppose that $c, k, l \in \mathbb{R}^{+}, c>l$ and $T, h: X_{\omega} \rightarrow X_{\omega}$ are two $\omega$-compatible mappings such that $T\left(X_{\omega}\right) \subseteq h\left(X_{\omega}\right)$ and

$$
\int_{0}^{\omega_{\frac{\lambda}{c}}(T x, T y)} \varphi(t) d t \leq k \int_{0}^{\omega_{\lambda}(h x, h y)} \varphi(t) d t
$$

for some $k \in(0,1)$ and for $\lambda>0$, where $\varphi: \mathbb{R}^{+} \rightarrow \mathbb{R}^{+}$is a Lebesgue integrable function which is summable, nonnegative and for all $\varepsilon>0$,

$$
\int_{0}^{\varepsilon} \varphi(t) d t>0
$$

If one of $T$ or $h$ is continuous, then there exists a unique common fixed point of $T$ and $h$.

Proof Let $x$ be an arbitrary point of $X_{\omega}$ and generate inductively the sequence $\left\{T x_{n}\right\}_{n=1}^{\infty}$ as follows: $T x_{n}=h x_{n+1}$ for each $n$ and $x_{1}=x$, that is possible as $T\left(X_{\omega}\right) \subseteq h\left(X_{\omega}\right)$. For each integer $n \geq 1$ and for all $\lambda>0$, (2.1) shows that

$$
\begin{aligned}
\int_{0}^{\omega_{\bar{\lambda}}\left(T x_{n+1}, T x_{n}\right)} \varphi(t) d t & \leq k \int_{0}^{\omega_{\frac{\lambda}{l}}\left(h x_{n+1}, h x_{n}\right)} \varphi(t) d t \\
& \leq k \int_{0}^{\omega_{\frac{\lambda}{c}}\left(T x_{n}, T x_{n-1}\right)} \varphi(t) d t \\
& \leq k^{2} \int_{0}^{\omega_{\lambda}\left(h x_{n}, h x_{n-1}\right)} \varphi(t) d t .
\end{aligned}
$$

By the principle of mathematical induction, we can easily show that

$$
\int_{0}^{\omega_{\frac{\lambda}{c}}\left(T x_{n+1}, T x_{n}\right)} \varphi(t) d t \leq k^{n} \int_{0}^{\omega_{\lambda}(T x, x)} \varphi(t) d t
$$

which, upon taking the limit as $n \rightarrow \infty$, yields

$$
\lim _{n \rightarrow \infty} \int_{0}^{\omega_{\frac{\lambda}{c}}\left(T x_{n+1}, T x_{n}\right)} \varphi(t) d t \leq 0 .
$$


Hence (2.2) implies that

$$
\lim _{n \rightarrow \infty} \omega_{\frac{\lambda}{c}}\left(T x_{n+1}, T x_{n}\right)=0
$$

We now show that $\left\{T x_{n}\right\}_{n=1}^{\infty}$ is Cauchy. So, for all $\varepsilon>0$, there exists $n_{0} \in \mathbb{N}$ such that $\omega_{\frac{\lambda}{c}}\left(T x_{n+1}, T x_{n}\right)<\frac{\varepsilon}{c}$ for all $n \in \mathbb{N}$ with $n \geq n_{0}$ and $\lambda>0$. Without loss of generality, suppose $m, n \in \mathbb{N}$ and $m>n$. Observe that for $\frac{\lambda}{c(m-n)}>0$, there exists $n_{\frac{\lambda}{m-n}} \in \mathbb{N}$ such that

$$
\omega_{\frac{\lambda}{c(m-n)}}\left(T x_{n+1}, T x_{n}\right)<\frac{\varepsilon}{c(m-n)}
$$

for all $n \geq n_{\frac{\lambda}{m-n}}$. We thus obtain

$$
\begin{aligned}
& \omega_{\frac{\lambda}{c}}\left(T x_{n}, T x_{m}\right) \\
& \quad \leq \omega_{\frac{\lambda}{c(m-n)}}\left(T x_{n}, T x_{n+1}\right)+\omega_{\frac{\lambda}{c(m-n)}}\left(T x_{n+1}, T x_{n+2}\right)+\cdots+\omega_{\frac{\lambda}{c(m-n)}}\left(T x_{m-1}, T x_{m}\right) \\
& \quad<\frac{\varepsilon}{c(m-n)}+\frac{\varepsilon}{c(m-n)}+\cdots+\frac{\varepsilon}{c(m-n)} \\
& \quad=\frac{\varepsilon}{c}
\end{aligned}
$$

for all $n, m \geq n_{\frac{\lambda}{m-n}}$. This implies that $\left\{T x_{n}\right\}_{n=1}^{\infty}$ is a Cauchy sequence. Since $X_{\omega}$ is complete, there exists $z \in X_{\omega}$ such that $\omega_{\frac{\lambda}{c}}\left(T x_{n}, z\right) \rightarrow 0$ as $n \rightarrow \infty$. If $T$ is continuous, then $T^{2} x_{n} \rightarrow T z$ and $T h x_{n} \rightarrow T z$. By the $\omega$-compatibility of $X_{\omega}$, we have $\omega_{\lambda}\left(h T x_{n}, T h x_{n}\right) \rightarrow 0$ as $n \rightarrow \infty$ for $\lambda>0$. Moreover, $h T x_{n} \rightarrow T z$ since $\omega_{\lambda}\left(h T x_{n}, T z\right) \leq \omega_{\frac{\lambda}{2}}\left(h T x_{n}, T h x_{n}\right)+\omega_{\frac{\lambda}{2}}\left(T h x_{n}, T z\right)$.

In the sequel, we prove that $z$ is a common fixed point of $T$ and $h$. By (2.1), we get

$$
\int_{0}^{\omega_{\frac{\lambda}{c}}\left(T^{2} x_{n}, T x_{n}\right)} \varphi(t) d t \leq k \int_{0}^{\omega_{\lambda}} \frac{\lambda^{l}}{\left(h T x_{n}, h x_{n}\right)} \varphi(t) d t .
$$

Taking the limit as $n \rightarrow \infty$ yields

$$
\begin{aligned}
\int_{0}^{\omega_{\frac{\lambda}{c}}(T z, z)} \varphi(t) d t & \leq k \int_{0}^{\omega_{\lambda}(T z, z)} \varphi(t) d t \\
& \leq k \int_{0}^{\omega_{\frac{\lambda}{c}}(T z, z)} \varphi(t) d t
\end{aligned}
$$

which implies that $\omega_{\frac{\lambda}{c}}(T z, z)=0$ for $\lambda>0$. Hence $T z=z$. It follows from $T\left(X_{\omega}\right) \subseteq h\left(X_{\omega}\right)$ that there exists a point $z_{1}$ such that $z=T z=h z_{1}$. By (2.1), we get

$$
\int_{0}^{\omega_{\frac{\lambda}{c}}\left(T^{2} x_{n}, T z_{1}\right)} \varphi(t) d t \leq k \int_{0}^{\omega_{\lambda}\left(h T x_{n}, h z_{1}\right)} \varphi(t) d t .
$$

Taking the limit as $n \rightarrow \infty$ yields

$$
\int_{0}^{\omega_{\frac{\lambda}{c}}\left(T z, T z_{1}\right)} \varphi(t) d t \leq k \int_{0}^{\omega_{\lambda}\left(T z, h z_{1}\right)} \varphi(t) d t,
$$


and so

$$
\begin{aligned}
\int_{0}^{\omega_{\frac{\lambda}{c}}\left(z, T z_{1}\right)} \varphi(t) d t & \leq k \int_{0}^{\omega_{\lambda}\left(z, h z_{1}\right)} \varphi(t) d t \\
& \leq k \int_{0}^{\omega_{\frac{\lambda}{l}}(z, z)} \varphi(t) d t .
\end{aligned}
$$

Hence $z=T z_{1}=h z_{1}$ and also $h z=h T z_{1}=T h z_{1}=T z=z$ (see [11]). In addition, if one considers $h$ to be continuous (instead of $T$ ), then by a similar argument (as above), one can prove $h z=T z=z$.

Finally, suppose that $z$ and $\omega$ are two arbitrary common fixed points of $T$ and $h$. Then we have

$$
\begin{aligned}
& \int_{0}^{\omega_{\frac{\lambda}{c}}(z, \omega)} \varphi(t) d t=\int_{0}^{\omega_{\frac{\lambda}{c}}(T z, T \omega)} \varphi(t) d t \\
& \leq k \int_{0}^{\omega_{\lambda}(h z, h \omega)} \varphi(t) d t \\
& \leq k \int_{0}^{\omega_{\frac{\lambda}{c}}(z, \omega)} \varphi(t) d t,
\end{aligned}
$$

which implies that $\omega_{\frac{\lambda}{c}}(z, \omega)=0$ for $\lambda>0$ and hence $z=\omega$.

The following theorem is another version of Theorem 2.2 when $l=c$, by adding the restriction that $T, h: B \rightarrow B$, where $B$ is a closed and bounded subset of $X_{\omega}$.

Theorem 2.3 Let $X_{\omega}$ be a complete modular metric space, and let $B$ be a closed and bounded subset of $X_{\omega}$. Suppose that $T, h: X_{\omega} \rightarrow X_{\omega}$ are two $\omega$-compatible mappings such that $T\left(X_{\omega}\right) \subseteq h\left(X_{\omega}\right)$ and

$$
\int_{0}^{\omega_{\frac{\lambda}{c}}(T x, T y)} \varphi(t) d t \leq k \int_{0}^{\omega_{\frac{\lambda}{c}}(h x, h y)} \varphi(t) d t
$$

for all $x, y \in B$ and for $\lambda>0$, where $c, k \in \mathbb{R}^{+}$with $k \in(0,1)$, and $\varphi: \mathbb{R}^{+} \rightarrow \mathbb{R}^{+}$is a Lebesgue integrable function which is summable, nonnegative and for all $\varepsilon>0, \int_{0}^{\varepsilon} \varphi(t) d t>0$. If one of $T$ or $h$ is continuous, then $T$ and $h$ have a unique common fixed point.

Proof Let $x \in B$ and $m, n \in \mathbb{N}$. Let $\left\{x_{n}\right\}_{n=1}^{\infty}$ be the sequence generated in the proof of Theorem 2.2. Then

$$
\begin{aligned}
& \int_{0}^{\omega_{\frac{\lambda}{c}}\left(T x_{n+m}, T x_{m}\right)} \varphi(t) d t \leq k \int_{0}^{\omega_{\frac{\lambda}{c}}\left(h x_{n+m}, h x_{m}\right)} \varphi(t) d t \\
& =k \int_{0}^{\omega_{\frac{\lambda}{c}}\left(T x_{n+m-1}, T x_{m-1}\right)} \varphi(t) d t \\
& \leq k^{2} \int_{0}^{\omega_{\frac{\lambda}{c}}\left(T x_{n+m-2}, T x_{m-2}\right)} \varphi(t) d t
\end{aligned}
$$




$$
\begin{aligned}
& \leq k^{m} \int_{0}^{\omega_{\frac{\lambda}{c}}\left(T x_{n}, x\right)} \varphi(t) d t \\
& \leq k^{m} \int_{0}^{\delta_{\omega}(B)} \varphi(t) d t
\end{aligned}
$$

for $\lambda>0$. Since $B$ is bounded,

$$
\lim _{n, m \rightarrow \infty} \int_{0}^{\omega_{\frac{\lambda}{c}}\left(T x_{n+m}, T x_{m}\right)} \varphi(t) d t \leq 0
$$

which implies that $\lim _{n, m \rightarrow \infty} \omega_{\frac{\lambda}{c}}\left(T x_{n+m}, T x_{m}\right)=0$. Therefore, $\left\{T x_{n}\right\}_{n=1}^{\infty}$ is Cauchy. Since $X_{\omega}$ is complete and $B$ is closed, there exists $z \in B$ such that $\lim _{n \rightarrow \infty} \omega_{\frac{\lambda}{c}}\left(T x_{n}, z\right)=0$. If $T$ is continuous, then $T^{2} x_{n} \rightarrow T z$ and $T h x_{n} \rightarrow T z$. Then, by $\omega$-compatibility of $X_{\omega}$, we have $\omega_{\lambda}\left(h T x_{n}, T h x_{n}\right) \rightarrow 0$ as $n \rightarrow \infty$ for $\lambda>0$. Moreover, $h T x_{n} \rightarrow T z$. Next, we prove that $z$ is a fixed point of $T$. It follows from (2.3) that

$$
\int_{0}^{\omega_{\frac{\lambda}{c}}\left(T^{2} x_{n}, T x_{n}\right)} \varphi(t) d t \leq k \int_{0}^{\omega_{\frac{\lambda}{c}}\left(h T x_{n}, h x_{n}\right)} \varphi(t) d t .
$$

Taking the limit as $n \rightarrow \infty$ yields

$$
\int_{0}^{\omega_{\frac{\lambda}{c}}(T z, z)} \varphi(t) d t \leq k \int_{0}^{\omega_{\frac{\lambda}{c}}(T z, z)} \varphi(t) d t .
$$

So $\omega_{\frac{\lambda}{c}}(T z, z)=0$ for $\lambda>0$ and hence $T z=z$. Since $T\left(X_{\omega}\right) \subseteq h\left(X_{\omega}\right)$, there exists a point $z_{1}$ such that $z=T z=h z_{1}$, and

$$
\int_{0}^{\omega} \frac{\lambda}{c}\left(T^{2} x_{n}, T z_{1}\right) \varphi(t) d t \leq k \int_{0}^{\omega_{\frac{\lambda}{c}}\left(h T x_{n}, h z_{1}\right)} \varphi(t) d t .
$$

Taking the limit as $n \rightarrow \infty$ yields

$$
\int_{0}^{\omega_{\frac{\lambda}{c}}\left(z, T z_{1}\right)} \varphi(t) d t \leq k \int_{0}^{\omega_{\frac{\lambda}{c}}(z, z)} \varphi(t) d t .
$$

Hence $z=T z_{1}=h z_{1}$ and also $h z=h T z_{1}=T h z_{1}=T z=z$ (see [11]). In addition, if one considers $h$ to be continuous (instead of $T$ ), then by a similar argument (as above), one can prove $h z=T z=z$.

Let $z$ and $\omega$ be two arbitrary common fixed points of $T$ and $h$. Then

$$
\begin{aligned}
\int_{0}^{\omega_{\frac{\lambda}{c}}(z, \omega)} \varphi(t) d t & =\int_{0}^{\omega_{\frac{\lambda}{c}}(T z, T \omega)} \varphi(t) d t \\
& \leq k \int_{0}^{\omega_{\frac{\lambda}{c}}(z, \omega)} \varphi(t) d t
\end{aligned}
$$

which implies that $\omega_{\frac{\lambda}{c}}(z, \omega)=0$ for $\lambda>0$ and hence $z=\omega$.

\section{A common fixed point theorem for quasi-contraction maps}

In this section, we prove Theorem 2.2 for a quasi-contraction map of integral type. To this end, we present the following definition. 
Definition 3.1 Two self-mappings $T, h: X_{\omega} \rightarrow X_{\omega}$ of a modular metric space $X_{\omega}$ are $(c, l, q)$-generalized contractions of integral type if there exist $0<q<1$ and $c, l \in \mathbb{R}^{+}$with $c>l$ such that

$$
\int_{0}^{\omega_{\frac{\lambda}{c}}(T x, T y)} \varphi(t) d t \leq q \int_{0}^{m(x, y)} \varphi(t) d t
$$

where $m(x, y)=\max \left\{\omega_{\frac{\lambda}{l}}(h x, h y), \omega_{\frac{\lambda}{l}}(h x, T x), \omega_{\frac{\lambda}{l}}(h y, T y), \frac{\omega_{l}(h x, T y)+\omega_{\frac{\lambda}{l}}(h y, T x)}{2}\right\}$, and $\varphi: \mathbb{R}^{+} \rightarrow$ $\mathbb{R}^{+}$is a Lebesgue integrable function which is summable, nonnegative and for all $\varepsilon>0$, $\int_{0}^{\varepsilon} \varphi(t) d t>0, \lambda>0$ and $x, y \in X_{\omega}$.

Theorem 3.2 Let $X_{\omega}$ be a complete modular metric space. Suppose that $T$ and $h$ are $(c, l, q)$-generalized contractions of integral type self-maps of $X_{\omega}$ and $T\left(X_{\omega}\right) \subseteq h\left(X_{\omega}\right)$. If one of $T$ or $h$ is continuous, then $T$ and $h$ have a unique common fixed point.

Proof Choose $c>2 l$. Let $x$ be an arbitrary point of $X_{\omega}$ and generate inductively the sequence $\left\{T x_{n}\right\}_{n=1}^{\infty}$ as follows: $T x_{n}=h x_{n+1}$ and $T\left(X_{\omega}\right) \subseteq h\left(X_{\omega}\right)$. We thus obtain

$$
\int_{0}^{\omega_{\frac{\lambda}{c}}\left(T x_{n+1}, T x_{n}\right)} \varphi(t) d t \leq q \int_{0}^{m\left(x_{n+1}, x_{n}\right)} \varphi(t) d t
$$

for $\lambda>0$, where

$$
\begin{aligned}
m\left(x_{n+1}, x_{n}\right)= & \max \left\{\omega_{\frac{\lambda}{l}}\left(h x_{n+1}, h x_{n}\right), \omega_{\frac{\lambda}{l}}\left(T x_{n}, h x_{n}\right), \omega_{\frac{\lambda}{l}}\left(h x_{n+1}, T x_{n+1}\right),\right. \\
& \left.\frac{\omega_{\frac{\lambda}{l}}\left(h x_{n+1}, T x_{n}\right)+\omega_{\frac{\lambda}{l}}\left(h x_{n}, T x_{n+1}\right)}{2}\right\} .
\end{aligned}
$$

It follows from $T x_{n}=h x_{n+1}$ that

$$
m\left(x_{n+1}, x_{n}\right)=\max \left\{\omega_{\frac{\lambda}{l}}\left(h x_{n+1}, h x_{n}\right), \omega_{\frac{\lambda}{l}}\left(T x_{n}, T x_{n+1}\right), \frac{0+\omega_{\frac{\lambda}{l}}\left(h x_{n}, T x_{n+1}\right)}{2}\right\} .
$$

Moreover,

$$
\begin{aligned}
\omega_{\frac{\lambda}{l}}\left(h x_{n}, T x_{n+1}\right) & =\omega_{\frac{\lambda}{l}}\left(T x_{n-1}, T x_{n+1}\right) \\
& \leq \omega_{\frac{\lambda}{2 l}}\left(T x_{n-1}, T x_{n}\right)+\omega_{\frac{\lambda}{2 l}}\left(T x_{n}, T x_{n+1}\right) \\
& \leq \omega_{\frac{\lambda}{c}}\left(T x_{n-1}, T x_{n}\right)+\omega_{\frac{\lambda}{c}}\left(T x_{n}, T x_{n+1}\right) .
\end{aligned}
$$

Then

$$
m\left(x_{n+1}, x_{n}\right) \leq \omega_{\frac{\lambda}{c}}\left(T x_{n}, T x_{n-1}\right)
$$

and

$$
\int_{0}^{\omega_{\frac{\lambda}{c}}\left(T x_{n+1}, T x_{n}\right)} \varphi(t) d t \leq q \int_{0}^{\omega_{\frac{\lambda}{c}}\left(T x_{n}, T x_{n-1}\right)} \varphi(t) d t .
$$


Continuing this process, we get

$$
\int_{0}^{\omega_{\frac{\lambda}{c}}\left(T x_{n+1}, T x_{n}\right)} \varphi(t) d t \leq q^{n} \int_{0}^{\omega_{\frac{\lambda}{c}}(T x, x)} \varphi(t) d t .
$$

So $\lim _{n \rightarrow \infty} \omega_{\frac{\lambda}{c}}\left(T x_{n}, T x_{n+1}\right)=0$ as $n$ tends to infinity. Suppose $l<c^{\prime}<2 l$. Since $\omega_{\lambda}$ is a decreasing function, one may write $\omega_{\frac{\lambda}{c^{\prime}}}\left(T x_{n}, T x_{n+1}\right) \leq \omega_{\frac{\lambda}{c}}\left(T x_{n}, T x_{n+1}\right)$, whenever $c^{\prime}<2 l \leq c$. Taking the limit from both sides of this inequality shows that $\lim _{n \rightarrow \infty} \omega_{\frac{\lambda}{c^{\prime}}}\left(T x_{n}, T x_{n+1}\right)=0$ for $l<c^{\prime}<2 l$ and $\lambda>0$. Thus we have $\lim _{n \rightarrow \infty} \omega_{\frac{\lambda}{c}}\left(T x_{n}, T x_{n+1}\right)=0$ for any $c>l$. Now, we show that $\left\{T x_{n}\right\}_{n=1}^{\infty}$ is Cauchy. Since $\lim _{n \rightarrow \infty} \omega_{\frac{\lambda}{c}}\left(T x_{n}, T x_{n+1}\right)=0$ for $\lambda>0$, for $\varepsilon>0$, there exists $n_{0} \in \mathbb{N}$ such that $\omega_{\frac{\lambda}{c}}\left(T x_{n+1}, T x_{n}\right)<\frac{\varepsilon}{c}$ for all $n \in \mathbb{N}$ with $n \geq n_{0}$ and $\lambda>0$. Without loss of generality, suppose $m, n \in \mathbb{N}$ and $m>n$. Observe that for $\frac{\lambda}{c(m-n)}>0$, there exists $n_{\frac{\lambda}{m-n}} \in \mathbb{N}$ such that

$$
\omega_{\frac{\lambda}{c(m-n)}}\left(T x_{n+1}, T x_{n}\right)<\frac{\varepsilon}{c(m-n)}
$$

for all $n \geq n_{\frac{\lambda}{m-n}}$. Now we have

$$
\begin{aligned}
& \omega_{\frac{\lambda}{c}}\left(T x_{n}, T x_{m}\right) \\
& \quad \leq \omega_{\frac{\lambda}{c(m-n)}}\left(T x_{n}, T x_{n+1}\right)+\omega_{\frac{\lambda}{c(m-n)}}\left(T x_{n+1}, T x_{n+2}\right)+\cdots+\omega_{\frac{\lambda}{c(m-n)}}\left(T x_{m-1}, T x_{m}\right) \\
& \quad<\frac{\varepsilon}{c(m-n)}+\frac{\varepsilon}{c(m-n)}+\cdots+\frac{\varepsilon}{c(m-n)} \\
& \quad=\frac{\varepsilon}{c}
\end{aligned}
$$

for all $n, m \geq n_{\frac{\lambda}{m-n}}$. This implies $\left\{T x_{n}\right\}_{n=1}^{\infty}$ is a Cauchy sequence. Since $X_{\omega}$ is complete, there exists $z \in X_{\omega}$ such that $\omega_{\frac{\lambda}{c}}\left(T x_{n}, z\right) \rightarrow 0$ as $n \rightarrow \infty$. Next we prove that $z$ is a fixed point of $T$. If $T$ is continuous, then $T^{2} x_{n} \rightarrow T z$ and $T h x_{n} \rightarrow T z$. By the $\omega$-compatibility of $X_{\omega}$, we have $\omega_{\lambda}\left(h T x_{n}, T h x_{n}\right) \rightarrow 0$ as $n \rightarrow \infty$ for $\lambda>0$. Moreover, $h T x_{n} \rightarrow T z$ since $\omega_{\lambda}\left(h T x_{n}, T z\right) \leq$ $\omega_{\frac{\lambda}{2}}\left(h T x_{n}, T h x_{n}\right)+\omega_{\frac{\lambda}{2}}\left(T h x_{n}, T z\right)$. Note that

$$
\int_{0}^{\omega_{\frac{\lambda}{c}}\left(T x_{n}, T^{2} x_{n}\right)} \varphi(t) d t \leq q \int_{0}^{m\left(x_{n}, T x_{n}\right)} \varphi(t) d t
$$

where

$$
\begin{aligned}
m\left(x_{n}, T x_{n}\right)= & \max \left\{\omega_{\frac{\lambda}{l}}\left(h x_{n}, h T x_{n}\right), \omega_{\frac{\lambda}{l}}\left(h x_{n}, T x_{n}\right), \omega_{\frac{\lambda}{l}}\left(h T x_{n}, T T x_{n}\right),\right. \\
& \left.\frac{\omega_{\frac{\lambda}{l}}\left(h x_{n}, T T x_{n}\right)+\omega_{\frac{\lambda}{l}}\left(T x_{n}, h T x_{n}\right)}{2}\right\} .
\end{aligned}
$$

Taking the limit as $n \rightarrow \infty$, we get

$$
\int_{0}^{\omega_{\frac{\lambda}{c}}(z, T z)} \varphi(t) d t \leq q \int_{0}^{\omega_{\frac{\lambda}{c}}(z, T z)} \varphi(t) d t
$$


and so $T z=z$. Since $T\left(X_{\omega}\right) \subseteq h\left(X_{\omega}\right)$, there exists a point $z_{1}$ such that $z=T z=h z_{1}$. We have

$$
\int_{0}^{\omega_{\frac{\lambda}{c}}\left(T^{2} x_{n}, T z_{1}\right)} \varphi(t) d t \leq q \int_{0}^{m\left(T x_{n}, z_{1}\right)} \varphi(t) d t
$$

and

$$
\begin{aligned}
m\left(T x_{n}, z_{1}\right)= & \max \left\{\omega_{\frac{\lambda}{l}}\left(h T x_{n}, z\right), \omega_{\frac{\lambda}{l}}\left(h T x_{n}, T^{2} x_{n}\right), \omega_{\frac{\lambda}{l}}\left(z, T z_{1}\right),\right. \\
& \left.\frac{\omega_{\frac{\lambda}{l}}\left(h T x_{n}, T z_{1}\right)+\omega_{\frac{\lambda}{l}}\left(z, T^{2} x_{n}\right)}{2}\right\} .
\end{aligned}
$$

Taking the limit as $n \rightarrow \infty$, we get

$$
\int_{0}^{\omega_{\frac{\lambda}{c}}\left(z, T z_{1}\right)} \varphi(t) d t \leq q \int_{0}^{\omega_{\frac{\lambda}{c}}\left(z, T z_{1}\right)} \varphi(t) d t
$$

It follows that $z=T z_{1}=h z_{1}$ and also $h z=h T z_{1}=T h z_{1}=T z=z$ (see [11]). Moreover, if $h$ is continuous (instead of $T$ ), then by a similar argument (as above), we can prove $h z=T z=z$. Let $z$ and $\omega$ be two arbitrary fixed points of $T$ and $h$. Then

$$
\begin{aligned}
m(z, \omega) & =\max \left\{\omega_{\frac{\lambda}{l}}(z, \omega), 0,0, \frac{\omega_{\frac{\lambda}{l}}(z, \omega)+\omega_{\frac{\lambda}{l}}(z, \omega)}{2}\right\} \\
& =\omega_{\frac{\lambda}{l}}(z, \omega) .
\end{aligned}
$$

Therefore,

$$
\int_{0}^{\omega_{\frac{\lambda}{c}}(z, \omega)} \varphi(t) d t \leq q \int_{0}^{\omega_{\lambda}(z, \omega)} \varphi(t) d t
$$

which implies that $z=\omega$.

\section{Generalization}

Here, we extend the results of the last section. We need a general contractive inequality of integral type. Let $\mathbb{R}^{+}$be a set of nonnegative real numbers and consider $(*) \phi: \mathbb{R}^{+} \rightarrow \mathbb{R}^{+}$ as a nondecreasing and right-continuous function such that $\phi(t)<t$ for any $t>0$.

To prove the next theorem, we need the following lemma [12].

Lemma 4.1 Let $t>0 . \phi(t)<$ t if only if $\lim _{k} \phi^{k}(t)=0$, where $\phi^{k}$ denotes the $k$-times repeated composition of $\phi$ with itself.

Next, we prove a modified version of Theorem 2.2.

Theorem 4.2 Let $X_{\omega}$ be a complete modular metric space. Suppose that $c, l \in \mathbb{R}^{+}, c>l$ and $T, h: X_{\omega} \rightarrow X_{\omega}$ are two $\omega$-compatible mappings such that $T\left(X_{\omega}\right) \subseteq h\left(X_{\omega}\right)$ and

$$
\int_{0}^{\omega_{\frac{\lambda}{c}}(T x, T y)} \varphi(t) d t \leq \phi\left(\int_{0}^{\omega_{\lambda}(h x, h y)} \varphi(t) d t\right)
$$


where $\phi$ is a function satisfying the property $(*)$, and $\varphi: \mathbb{R}^{+} \rightarrow \mathbb{R}^{+}$is a Lebesgue integrable mapping which is summable, nonnegative and for all $\varepsilon>0, \int_{0}^{\varepsilon} \varphi(t) d t>0$ and $\lambda>0$. If one of $T$ or $h$ is continuous, then $T$ and $h$ have a unique common fixed point.

Proof Let $x$ be an arbitrary point of $X_{\omega}$ and generate inductively the sequence $\left\{T x_{n}\right\}_{n=1}^{\infty}$ as follows: $T x_{n}=h x_{n+1}$ and $x_{1}=x$, that is possible as $T\left(X_{\omega}\right) \subseteq h\left(X_{\omega}\right)$,

$$
\begin{aligned}
\int_{0}^{\omega_{\frac{\lambda}{c}}\left(T x_{n+1}, T x_{n}\right)} \varphi(t) d t & \leq \phi\left(\int_{0}^{\omega_{\frac{\lambda}{l}}\left(h x_{n+1}, h x_{n}\right)} \varphi(t) d t\right) \\
& \leq \phi\left(\int_{0}^{\omega_{\frac{\lambda}{c}}\left(T x_{n}, T x_{n-1}\right)} \varphi(t) d t\right) \\
& \leq \phi^{2}\left(\int_{0}^{\omega_{\lambda}\left(h x_{n}, h x_{n-1}\right)} \varphi(t) d t\right)
\end{aligned}
$$

for each integer $n \geq 1$ and $\lambda>0$. By the principle of mathematical induction, we can easily see that

$$
\int_{0}^{\omega_{\frac{\lambda}{c}}\left(T x_{n+1}, T x_{n}\right)} \varphi(t) d t \leq \phi^{n}\left(\int_{0}^{\omega_{\lambda}(T x, x)} \varphi(t) d t\right) .
$$

Taking the limit as $n \rightarrow \infty$, we obtain, by Lemma 4.1,

$$
\lim _{n} \int_{0}^{\omega_{\frac{\lambda}{c}}\left(T x_{n+1}, T x_{n}\right)} \varphi(t) d t=0
$$

Using the same method as in the proof of Theorem 2.2, we show that $T$ and $h$ have a unique common fixed point.

Applying the method of proof of Theorem 3.2, we get the following result.

Theorem 4.3 Let $X_{\omega}$ be a complete modular metric space. Suppose $c, l \in \mathbb{R}^{+}, c>l$ and $T, h: X_{\omega} \rightarrow X_{\omega}$ such that $T\left(X_{\omega}\right) \subseteq h\left(X_{\omega}\right)$

$$
\int_{0}^{\omega_{\frac{\lambda}{c}}(T x, T y)} \varphi(t) d t \leq \phi\left(\int_{0}^{m(x, y)} \varphi(t) d t\right)
$$

where $m(x, y)=\max \left\{\omega_{\frac{\lambda}{l}}(h x, h y), \omega_{\lambda}(h x, T x), \omega_{\lambda}(h y, T y), \frac{\omega_{\lambda}(h x, T y)+\omega_{\lambda}(h y, T x)}{2}\right\}, \phi$ is a function satisfying the property $(*)$ and $\lambda>0$. If one of $T$ or $h$ is continuous, then there exists a unique common fixed point of $h$ and $T$.

Proof The proof is similar to the proof of Theorem 3.2.

Now we provide examples to validate and illustrate Theorems 2.2 and 3.2.

Example 4.4 Let $X_{\omega}=\left\{\frac{1}{n}: n \in \mathbf{N}\right\} \cup\{0\}$ and $\omega_{\lambda}(x, y):=\frac{|x-y|}{\lambda}$ for $\lambda>0$. Define the mapping $T, h: X_{\omega} \rightarrow X_{\omega}$ by

$$
T(x):=\left\{\begin{array}{ll}
\frac{1}{n+4} & \text { if } x=\frac{1}{n}, \\
0 & \text { otherwise }
\end{array} \text { and } \quad h(x):= \begin{cases}\frac{1}{n+1} & \text { if } x=\frac{1}{n}, \\
0 & \text { otherwise }\end{cases}\right.
$$


Then all the hypotheses of Theorem 2.2 are satisfied with $\varphi(t)=2 t$ for $t>0$ and $c=2, l=1$, $k=\frac{1}{4}$.

Example 4.5 Let $X_{\omega}=\left\{\frac{1}{n}: n \in \mathbf{N}\right\} \cup\{0\}$ and $\omega_{\lambda}(x, y):=\frac{|x-y|}{\lambda}$ for $\lambda>0$. Define the mapping $T, h: X_{\omega} \rightarrow X_{\omega}$ by

$$
T(x):=\left\{\begin{array}{ll}
\frac{1}{n+1} & \text { if } x=\frac{1}{n}, \\
0 & \text { otherwise }
\end{array} \quad \text { and } \quad h(x):= \begin{cases}\frac{1}{n} & \text { if } x=\frac{1}{n} \\
0 & \text { otherwise }\end{cases}\right.
$$

Then all the hypotheses of Theorem 3.2 are satisfied with $\varphi(t)=t^{\frac{1}{t-2}}(1-\log t)$ for $t>0$ and $c=l=\lambda=1, k=\frac{1}{2}$. See [4] for details.

\section{Application}

In the section, we assume that $\mathbb{R}=(-\infty,+\infty), \mathbb{R}^{+}=(0,+\infty), \mathbb{N}$ denotes the set of all positive integers, 'opt' stands for 'sup' or 'inf', $Y$ is a Banach space and $X_{\omega}$ is an $\omega$-complete space. Suppose that $S \subseteq X_{\omega}, D \subseteq Y$ and $B(S)$ denotes the complete space of all bounded real-valued functions on $S$ with the norm

$$
\|g\|=\sup \{|g(x)|: x \in S\} \quad(g \in B(S))
$$

and $\Phi=\left\{\varphi ; \varphi: \mathbb{R}^{+} \rightarrow \mathbb{R}^{+}\right\}$such that $\varphi$ is Lebesgue integrable, summable on each compact subset of $\mathbb{R}^{+}$and $\int_{0}^{\varepsilon} \varphi(t) d t>0$ for each $\varepsilon>0$. We prove the solvability of the functional equations

$$
f(x)=\underset{y \in D}{\operatorname{opt}}\{u(x, y)+H(x, y, f(T(x, y)))\}
$$

for all $x \in S$ in $B(S)$. First, we recall the following lemma [13].

Lemma 5.1 ([13]) Let $E$ be a set, and let $p, q: E \rightarrow \mathbb{R}$ be mappings. If opt $_{y \in E} p(y)$ and opt $_{y \in E} q(y)$ are bounded, then

$$
\left|\operatorname{opt}_{y \in E} p(t)-\underset{y \in E}{\operatorname{opt}} q(t)\right| \leq \sup _{y \in E}|p(y)-q(y)|
$$

Theorem 5.2 Let u $S \times D \rightarrow \mathbb{R}, T: S \times D \rightarrow S, H: S \times D \times \mathbb{R} \rightarrow \mathbb{R}, \phi \in \Phi$. Suppose that $u$ and $H$ are bounded such that

$$
\int_{0}^{\frac{c|H(x, y, g(T(x, y)))-H(x, y, h(T(x, y)))|}{\lambda}} \varphi(t) d t \leq k \int_{0}^{\frac{l\|g-h\|}{\lambda}} \varphi(t) d t
$$

for all $(x, y, g, h, \lambda) \in S \times D \times B(S) \times B(S) \times \mathbb{R}^{+}$, and some $0<k<1$ and $c>l>0$. Then the functional equation (5.1) has a unique solution $w \in B(S)$ and $\left\{A^{n} z\right\}_{n \in \mathbb{N}}$ converges to $w$ for each $z \in B(S)$, where the mapping $A$ is defined by

$$
A z(x)=\operatorname{opt}_{y \in D}\{u(x, y)+H(x, y, z(T(x, y)))\} \quad(x \in S) .
$$


Proof By boundedness of $u$ and $H$, there exists $M>0$ such that

$$
\sup \{|u(x, y)|+|H(x, y, z(T(x, y)))|:(x, y, t) \in S \times D \times \mathbb{R}\} \leq M
$$

It is easy to show that $A$ is a self-mapping in $B(S)$ by (5.3), (5.4) and Lemma 5.1. Using [14, Theorem 12.34] and $\phi \in \Phi$, we conclude that for each $\varepsilon>0$, there exists $\delta>0$ such that

$$
\int_{C} \varphi(t) d t<\varepsilon, \quad \forall C \subseteq[0,2 M] \text { with } m(C) \leq \delta
$$

where $m(C)$ denotes the Lebesgue measure of $C$.

Let $x \in S, h, g \in B(S)$. Suppose that opt ${ }_{y \in D}=\inf _{y \in D}$. Clearly, for $c>l$, (5.3) implies that there exist $y, z \in D$ satisfying

$$
\begin{aligned}
& A g(x)>u(x, y)+H(x, y, g(T(x, y)))-\frac{\delta}{c} \\
& A h(x)>u(x, z)+H(x, z, h(T(x, z)))-\frac{\delta}{c} \\
& A g(x) \leq u(x, z)+H(x, z, g(T(x, z))) \\
& A h(x) \leq u(x, y)+H(x, y, h(T(x, y)))
\end{aligned}
$$

Put $H_{1}=H(x, y, g(T(x, y))), H_{2}=H(x, y, h(T(x, y))), H_{3}=H(x, z, g(T(x, z))), H_{4}=H(x, z$, $h(T(x, z)))$.

From (5.6) and (5.9), it follows that

$$
\begin{aligned}
c(A g(x)-A h(x))> & c(H(x, y, g(T(x, y)))-H(x, y, h(T(x, y))))-\delta \\
> & l(H(x, y, g(T(x, y)))-H(x, y, h(T(x, y))))-\delta \\
\geq & -\max \{l|H(x, y, g(T(x, y)))-H(x, y, h(T(x, y)))|, \\
& l|H(x, z, g(T(x, z)))-H(x, z, h(T(x, z)))|\}-\delta \\
= & -\max \left\{l\left|H_{1}-H_{2}\right|, l\left|H_{3}-H_{4}\right|\right\}-\delta .
\end{aligned}
$$

Similarly, from (5.7) and (5.8), we get

$$
\begin{aligned}
c(A h(x)-A g(x))> & c(H(x, z, h(T(x, z)))-H(x, z, g(T(x, z))))-\delta \\
> & l(H(x, z, h(T(x, z)))-H(x, z, g(T(x, z))))-\delta \\
\geq & -\max \{l|H(x, y, g(T(x, y)))-H(x, y, h(T(x, y)))|, \\
& l|H(x, z, g(T(x, z)))-H(x, z, h(T(x, z)))|\}-\delta \\
= & -\max \left\{l\left|H_{1}-H_{2}\right|, l\left|H_{3}-H_{4}\right|\right\}-\delta .
\end{aligned}
$$

So

$$
c(A g(x)-A h(x))<\max \left\{l\left|H_{1}-H_{2}\right|, l\left|H_{3}-H_{4}\right|\right\}+\delta .
$$


Then

$$
\frac{c|A g(x)-A h(x)|}{\lambda}<\max \left\{\frac{l\left|H_{1}-H_{2}\right|}{\lambda}, \frac{l\left|H_{3}-H_{4}\right|}{\lambda}\right\}+\frac{\delta}{\lambda}
$$

for each $\lambda>0$.

Similarly, we infer that (5.10) holds also for opt ${ }_{y \in D}=\sup _{y \in D}$. Combining (5.2), (5.5) and (5.10) yields

$$
\begin{aligned}
\int_{0}^{\frac{c|A g(x)-A h(x)|}{\lambda} \varphi(t) d t \leq} & \int_{0}^{\max \left\{\frac{l\left|H_{1}-H_{2}\right|}{\lambda}, \frac{l\left|H_{3}-H_{4}\right|}{\lambda}\right\}+\frac{\delta}{\lambda}} \varphi(t) d t \\
= & \max \left\{\int_{0}^{\frac{l\left|H_{1}-H_{2}\right|}{\lambda}+\frac{\delta}{\lambda}} \varphi(t) d t, \int_{0}^{\frac{l\left|H_{3}-H_{4}\right|}{\lambda}+\frac{\delta}{\lambda}} \varphi(t) d t\right\} \\
= & \max \left\{\int_{0}^{\frac{l\left|H_{1}-H_{2}\right|}{\lambda}} \varphi(t) d t+\int_{\frac{l\left|H_{1}-H_{2}\right|}{\lambda}}^{\frac{|| H_{1}-H_{2} \mid}{\lambda}+\frac{\delta}{\lambda}} \varphi(t) d t,\right. \\
& \left.\int_{0}^{\frac{l|| H_{3}-H_{4} \mid}{\lambda}} \varphi(t) d t+\int_{\frac{|| H_{3}-H_{4} \mid}{\lambda}}^{\frac{|l| H_{3}-H_{4} \mid}{\lambda}+\frac{\delta}{\lambda}} \varphi(t) d t\right\} \\
\leq & \max \left\{\int_{0}^{\frac{l\left|H_{1}-H_{2}\right|}{\lambda}} \varphi(t) d t, \int_{0}^{\frac{l\left|H_{3}-H_{4}\right|}{\lambda}} \varphi(t) d t\right\} \\
& +\max \left\{\int_{\frac{l\left|H_{1}-H_{2}\right|}{\lambda}}^{\frac{l\left|H_{1}-H_{2}\right|}{\lambda}+\frac{\delta}{\lambda}} \varphi(t) d t, \int_{\frac{l\left|H_{3}-H_{4}\right|}{\lambda}}^{\frac{l\left|H_{3}-H_{4}\right|}{\lambda}+\frac{\delta}{\lambda}} \varphi(t) d t\right\} \\
\leq & k \int_{0}^{\frac{l|| g-h \|}{\lambda}} \varphi(t) d t+\varepsilon,
\end{aligned}
$$

which means that

$$
\int_{0}^{\frac{c\|A g-A h\|}{\lambda}} \varphi(t) d t \leq k \int_{0}^{\frac{l\|g-h\|}{\lambda}} \varphi(t) d t+\varepsilon
$$

for each $\lambda>0$. Letting $\varepsilon \rightarrow 0^{+}$in the above inequality, we deduce that

$$
\int_{0}^{\frac{c\|A g-A h\|}{\lambda}} \varphi(t) d t \leq k \int_{0}^{\frac{l\|g-h\|}{\lambda}} \varphi(t) d t .
$$

Thus Theorem 5.2 follows from Theorem 2.2. This completes the proof.

\section{Competing interests}

The authors declare that they have no competing interests.

Authors' contributions

All authors conceived of the study, participated in its design and coordination, drafted the manuscript, participated in the sequence alignment, and read and approved the final manuscript.

\section{Author details}

'Department of Mathematics and Computer Sciences, Hakim Sabzevari University, P.O. Box 397, Sabzevar, Iran.

${ }^{2}$ Department of Mathematics, Iran University of Science and Technology, Tehran, Iran. ${ }^{3}$ Department of Mathematics,

Research Institute for Natural Sciences, Hanyang University, Seoul, 133-791, Korea. 


\section{Acknowledgements}

The authors are grateful to the two anonymous reviewers for their valuable comments and suggestions.

Received: 1 May 2013 Accepted: 17 September 2013 Published: 07 Nov 2013

\section{References}

1. Jungck, G: Commuting mappings and fixed point. Am. Math. Mon. 83, 261-263 (1976)

2. Sessa, S: On a weak commutativity conditions of mappings in fixed point consideration. Publ. Inst. Math. (Belgr.) 32, 146-153 (1982)

3. Jungck, G: Compatible mappings and common fixed points. Int. J. Math. Math. Sci. 11, 771-779 (1986)

4. Branciari, A: A fixed point theorem for mappings satisfying a general contractive condition of integral type. Int. J. Math. Math. Sci. 29, 531-536 (2002)

5. Samet, B, Vetro, C: An integral version of Ciric's fixed point theorem. Mediterr. J. Math. 9, 225-238 (2012)

6. Samet, B, Vetro, C: Berinde mappings in orbitally complete metric spaces. Chaos Solitons Fractals 44, 1075-1079 (2011)

7. Vetro, C: On Branciari's theorem for weakly compatible mappings. Appl. Math. Lett. 23, 700-705 (2010)

8. Vijayaraju, P, Rhoades, BE, Mohanra, R: A fixed point theorem for a pair of maps satisfying a general contractive condition of integral type. Int. J. Math. Math. Sci. 15, 2359-2364 (2005)

9. Razani, A, Moradi, R: Common fixed point theorems of integral type in modular spaces. Bull. Iran. Math. Soc. 35, 11-24 (2009)

10. Chistyakov, V: Modular metric spaces I: basic concepts. Nonlinear Anal. TMA 72, 1-14 (2010)

11. Kaneko, H, Sessa, S: Fixed point theorems for compatible multi-valued and single-valued mappings. Int. J. Math. Math. Sci. 12, 257-262 (1989)

12. Singh, SP, Meade, BA: On common fixed point theorem. Bull. Aust. Math. Soc. 16, $49-53$ (1977)

13. Liu, Z, Li, X, Kang, SM, Cho, SY: Fixed point theorems for mappings satisfying contractive conditions of integral type and applications. Fixed Point Theory Appl. 2011, 64 (2011)

14. Hewitt, E, Stromberg, K: Real and Abstract Analysis. Springer, Berlin (1978)

10.1186/1029-242X-2013-483

Cite this article as: Azadifar et al.: Integral type contractions in modular metric spaces. Journal of Inequalities and Applications 2013, 2013:483

\section{Submit your manuscript to a SpringerOpen ${ }^{\circ}$ journal and benefit from:}

- Convenient online submission

- Rigorous peer review

Immediate publication on acceptance

Open access: articles freely available online

- High visibility within the field

- Retaining the copyright to your article 